\title{
MANEJO QUÍMICO DE PLANTAS DANINHAS EM ÁREA DE PLANTIO DIRETO DE MANDIOCA
}

\author{
Rubem S. Oliveira Jr. ${ }^{1}$, Jamil Constantin ${ }^{1}$, Alexandre I. F. M. Hernandes ${ }^{2}$, Miriam H. Inoue ${ }^{3}$, \\ Orivaldo Marchiori Jr. ${ }^{3}$ e Antônio C. Ramires ${ }^{4}$
}

\footnotetext{
${ }^{1}$ Eng ${ }^{\circ}$. Agrônomo, Dr., Professor Adjunto. Universidade Estadual de Maringá. Av. Colombo, 5790. Maringá, PR $87020-900$ rsojunior@uem.br

${ }^{2}$ Acadêmico de Agronomia. Universidade Estadual de Maringá

${ }^{3}$ Estudante do Curso de Pós-graduação em Agronomia. Universidade Estadual de Maringá

${ }^{4}$ Eng ${ }^{\circ}$. Agrônomo. Universidade Estadual de Maringá
}

\section{RESUMO}

O objetivo do trabalho foi avaliar a seletividade para a cultura da mandioca e o comportamento de vários herbicidas, aplicados em pré e pós-emergência, no controle das plantas daninhas Avena sativa, Bidens pilosa, Commelina benghalensis, Raphanus sativus e Sida rhombifolia. O experimento foi conduzido em área de plantio direto, em Araruna, noroeste do Paraná. Embora alguns herbicidas avaliados tenham se destacado em relação ao controle de uma ou mais das espécies de plantas daninhas e outros tenham demonstrado seletividade para a mandioca, os melhores resultados foram obtidos, considerando-se tanto a seletividade quanto a eficácia, com as misturas ametryne+clomazone $(1,50+1,00 \mathrm{~kg} / \mathrm{ha})$ e ametryne+diuron $(0,62+0,96 \mathrm{~kg} / \mathrm{ha})$, ambas aplicadas em pós-emergência das plantas daninhas e quando a mandioca encontrava-se com 5 a $10 \%$ de brotações emergidas.

Palavras-chave: herbicidas, Manihot esculenta, seletividade.

\section{ABSTRACT \\ Chemical weed control in areas of no-till cassava}

The aim of this work was to evaluate the selectivity of some pre and postemergence herbicides on cassava, as well as their efficiency to control the weeds Avena sativa, Bidens pilosa, Commelina benghalensis, Raphanus sativus and Sida rhombifolia. The field trial was carried out in no-till areas, in Araruna, northwestern Paraná State. Although some herbicides provided outstanding results either in relation to weed control or crop selectivity, when both aspects were taken into consideration, the best results were achieved with ametryne+clomazone $(1.50+1.00 \mathrm{~kg} / \mathrm{ha})$ and ametryne+diuron $(0.62+0.96$ $\mathrm{kg} / \mathrm{ha}$ ), both applied postemergence of weeds, when 5 to $10 \%$ of cassava sprouts had already emerged from the soil.

Key words: herbicides, Manihot esculenta, selectivity. 


\section{INTRODUÇÃO}

A mandioca (Manihot esculenta) ocupa posição de destaque na agricultura como um dos alimentos energéticos mais consumidos nos trópicos, sendo suplantada apenas pelo arroz, cana-de-açúcar e milho (FAO, 1996). A parte economicamente mais importante da planta são as raízes tuberosas, ricas em amido, que são utilizadas na alimentação humana e animal ou como matéria-prima para diversas indústrias (alimentícia, farmacêutica, de papel, têxtil) (Peressin, 1997). No Brasil, a mandioca é cultivada em regiões ecologicamente diversas, adaptando-se a amplas variações de clima, principalmente quanto aos fatores temperatura, precipitação, fotoperíodo e intensidade luminosa (Alves, 1990).

Diversos autores têm procurado avaliar o período de competição entre a mandioca e as plantas daninhas que ocasiona as maiores perdas de produtividade. Waron \& Gartner (1972) afirmaram que a cultura deve permanecer livre de plantas daninhas durante os seis primeiros meses de seu desenvolvimento. Doll \& Piedrahita (1976) concluíram que as plantas infestantes da cultura da mandioca causam os maiores danos sobre o rendimento de raízes até que a parte aérea da cultura cubra o solo. Pinho et al. (1980) relatam que capinas realizadas até 90 dias após o plantio aumentam consideravelmente a produção de ramas e raízes da mandioca. Alcântara ét al. (1982) evidenciaram que a manutenção da cultura no limpo a partir de 120 dias após o plantio não incrementou significativamente a produção. Howeler \& Ballesteros (1987), no entanto, observaram que a mandioca é muito sensível à competição imposta pelas plantas infestantes durante os primeiros três a quatro meses de idade. Carvalho et al. $(1990,1993)$ recomendam que a mandioca deve permanecer sem matocompetição por um período de 90 a 120 dias após a emergência. Mais recentemente, Peressin (1997) observou, em média, reduções de 95 e $20 \%$ na produtividade de raízes tuberosas, respectivamente, para os experimentos conduzidos por um e por dois ciclos vegetativos que compararam as testemunhas no mato e no limpo. Em suma, a cultura é mais afetada pela interferência imposta pelas plantas daninhas durante os três ou quatro primeiros meses após o plantio.

A eliminação das invasoras representa atualmente cerca de $56 \%$ da mão-de-obra utilizada no plantio e condução da mandioca, o que significa aproximadamente $30 \%$ do custo total de produção (Miranda et al., 1995). A planta de mandioca apresenta como característica o lento crescimento inicial, o que leva o solo a ficar exposto às intempéries climáticas por período relativamente longo. Esta característica, associada ao fato de que o principal pólo mandioqueiro do Estado do Paraná situa-se na região noroeste, cujos solos são predominantemente arenosos, com baixos teores de carbono orgânico e, portanto, predispostos aos processos erosivos, tem suscitado o interesse no plantio direto para a mandioca na região. Além da redução na exposição do solo, o plantio direto pode trazer outros benefícios, tais como: valores mais ele- vados de matéria orgânica em relação aos demais sistemas de cultivo, redução populacional das plantas daninhas, diminuição das operações de preparo de solo e conseqüentemente do custo de produção (Ferreira \& Farias, 1997). Entretanto, o comportamento de herbicidas na presença da palha pode ser bastante alterado (Rodrigues, 1993; Fornarolli et al., 1998).

Pouco se sabe, portanto, a respeito da seletividade e eficácia de possíveis novas alternativas de controle químico de plantas daninhas na cultura da mandioca, implantada nestas condições. Doll \& Piedrahita $(1974,1976)$, Oliveira Jr. (1994) e Oliveira Jr. et al. (1995) indicam que a resposta da mandioca à aplicação de herbicidas varia desde a total seletividade até o completo comprometimento da produção, por causa da fitotoxicidade provocada à cultura. Em alguns casos, a variação na resposta da mandioca a herbicidas ocorre em função da dose aplicada (Alcântara \& Lima, 1982) ou tipo de solo no qual o trabalho foi conduzido (Alcântara \& Souza, 1982).

O presente trabalho teve como objetivos analisar a eficácia e seletividade de diversos herbicidas aplicados em área de plantio direto de mandioca, visando atender às necessidades da agroindústria da mandioca da região noroeste do Estado do Paraná.

\section{MATERIAL E MÉTODOS}

A pesquisa foi desenvolvida na Fazenda da Pinduca Indústria Alimentícia Ltda, em Araruna, Estado do Paraná. A característica climática do município é do tipo $\mathrm{Cfb}$ (conforme Köppen), com temperatura média anual de $21,5^{\circ} \mathrm{C}$ e precipitação média anual de $1617 \mathrm{~mm}$. O solo onde se instalou o experimento é classificado como Latossolo Vermelho distrófico, textura média, cujas características químicas e físicas encontram-se na Tabela 1 .

A área do experimento, que havia sido cultivada anteriormente com aveia, foi dessecada com glyphosate $(0,72 \mathrm{~g} /$ ha e.a.) 20 dias antes do plantio de mandioca. O preparo do solo consistiu apenas da utilização de rolo-faca sete dias antes do plantio, que foi realizado mecanicamente em 15/10/ 99, de modo a propiciar cobertura morta para o solo.

Foi utilizada a cultivar Espeto, cujas manivas, com tamanho médio de $15 \mathrm{~cm}$, foram plantadas horizontalmente nos sulcos, a aproximadamente $10 \mathrm{~cm}$ de profundidade, sendo que a primeira precipitação $(19,8 \mathrm{~mm})$ após o plantio ocorreu em 16/10/99. Os tratos culturais realizados foram aqueles normalmente empregados para a cultura, conforme Conceição (1979) e Lorenzi \& Dias (1993).

A aplicação dos herbicidas, em pré-emergência, foi realizada em 21/10/99 e em pós-emergência em 01/11/99, quando 5 a $10 \%$ das manivas estavam brotadas. As plantas daninhas encontravam-se todas com até 4 folhas. Na ocasião de ambas as aplicações o solo apresentava-se seco, com umidade relativa do ar superior a $80 \%$, ventos moderados e a 
Tabela 1. Características químicas e físicas de amostras de solo oriundas da área do experimento de campo. Araruna, PR. 1999/2000.

\begin{tabular}{|c|c|c|c|c|c|c|c|c|c|c|c|}
\hline $\mathrm{pH}$ & & $\mathrm{Al}^{3+}$ & $\mathrm{H}^{+}+\mathrm{Al}^{3+}$ & $\mathrm{Ca}^{2+}+\mathrm{Mg}^{2+}$ & $\mathrm{Ca}^{2+}$ & $\mathrm{K}^{+}$ & \multirow{2}{*}{$\begin{array}{c}\mathrm{P} \\
\mathrm{mg} / \mathrm{dm}^{3}\end{array}$} & \multirow{2}{*}{$\begin{array}{c}\mathrm{C} \\
\mathrm{g} / \mathrm{dm}^{3}\end{array}$} & Areia & Silte & \multirow[t]{2}{*}{ Argila } \\
\hline $\mathrm{CaCl}_{2}$ & $\mathrm{H}_{2} \mathrm{O}$ & ---- & 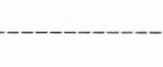 & $-\mathrm{cmol}_{\mathrm{c}} / \mathrm{dm}^{3}-$ & & --- & & & & -0 & \\
\hline 5,9 & 6,7 & 0,00 & 2,54 & 4,83 & 3,08 & 0,39 & 6,00 & 9,18 & 70 & 01 & 29 \\
\hline
\end{tabular}

temperatura variava de 18 a $20^{\circ} \mathrm{C}$. Foi utilizado um pulverizador costal de pressão constante a base de $\mathrm{CO}_{2}$, com bicos leque $110-\mathrm{SF}-02$, pressão de $2,0 \mathrm{~kg} / \mathrm{cm}^{2}$, o qual proporcionou um consumo de calda de $200 \mathrm{l} / \mathrm{ha}$. A testemunha capinada foi mantida limpa através de capinas manuais durante todo o ciclo da cultura. Na Tabela 2 encontram-se relacionados os tratamentos, modalidades de aplicação e doses utilizadas no experimento. Nos tratamentos em que apareceram dois princípios ativos foram utilizados herbicidas comerciais cujas composições são misturas formuladas. Após o período inicial de avaliação do efeito dos herbicidas sobre o controle das plantas daninhas (48 dias após o plantio), as parcelas tratadas com herbicidas e a testemunha sem capina foram capina- das até a colheita.

No momento da aplicação dos herbicidas em pós-emergência, as plantas daninhas presentes e respectivas densidades de infestação e estádios de desenvolvimento eram Avena sativa (20 plantas $/ \mathrm{m}^{2}, 10 \mathrm{~cm}$ de altura), Bidens pilosa (12 plantas $/ \mathrm{m}^{2}, 2$ a 4 folhas), Raphanus sativus (10 plantas $/ \mathrm{m}^{2}$, 2 a 3 folhas), Commelina benghalensis ( 3 plantas $/ \mathrm{m}^{2}, 2$ a 3 folhas), e Sida rhombifolia (2 plantas $/ \mathrm{m}^{2}, 1$ a 2 folhas). Apesar da baixa densidade de infestação destas duas últimas espécies, assim mesmo seus controles foram avaliados tendo em vista a distribuição uniforme das duas espécies na área experimental.

O delineamento experimental utilizado foi o de blocos

Tabela 2. Nomes comum e comercial dos herbicidas aplicados em pré (tratamentos 3 a 14) ou pós-emergência (tratamentos 14 a 22) e doses utilizadas no experimento. Araruna, PR. 1999/2000.

\begin{tabular}{|c|c|c|c|}
\hline \multicolumn{2}{|c|}{ Tratamento } & \multicolumn{2}{|c|}{ Dose } \\
\hline Nome comum & Nome comercial & i.a. ${ }^{3}$ (kg/ha) & p.c. $^{4}$ (l ou kg/ha) \\
\hline 1. Testemunha capinada & - & - & - \\
\hline 2. Testemunha sem capina & - & - & - \\
\hline 3. Acetochlor & Surpass & 3,072 & 4,001 \\
\hline 4. Alachlor & Alaclor Nortox & 2,000 & 4,171 \\
\hline 5. Ametryne & Herbipak & 1,500 & 3,001 \\
\hline 6. Ametryne/diuron & Ametron & $0,620+0,960$ & 4,001 \\
\hline 7. Flumioxazin & Flumyzin & 0,040 & $0,08 \mathrm{~kg}$ \\
\hline 8. Isoxaflutole & Provence & 0,075 & $0,10 \mathrm{~kg}$ \\
\hline 9. Metolachlor & Dual & 2,000 & 2,081 \\
\hline 10. Metolachlor/metribuzin & Corsum & $1,680+0,240$ & 2,001 \\
\hline 11. Metribuzin & Sencor & 0,480 & 1,001 \\
\hline 12. Oxyfluorfen & Galigan & 0,480 & 2,001 \\
\hline 13. Sulfentrazone & Boral & 0,600 & 1,201 \\
\hline 14. Trifluralin & Premerlin & 1,800 & 3,001 \\
\hline 15. Aclonifen & Prodigio & 0,900 & 1,501 \\
\hline 16. Ametryne $e^{1}$ & Herbipak & 1,500 & 3,001 \\
\hline 17. Ametryne/clomazone & Sinerge & $1,500+1,000$ & 5,001 \\
\hline 18. Ametryne/diuron ${ }^{1}$ & Ametron & $0,620+0,960$ & 4,001 \\
\hline 19. Flumiclorac-pentil & Radiant & 0,050 & 0,501 \\
\hline 20. Isoxaflutole ${ }^{2}$ & Provence & 0,038 & $0,05 \mathrm{~kg}$ \\
\hline 21. Oxasulfuron & Chart & 0,045 & $0,06 \mathrm{~kg}$ \\
\hline 22. Oxyfluorfen & Galigan & 0,480 & 2,001 \\
\hline
\end{tabular}

${ }^{1}$ Foi utilizado óleo mineral (Agral $\left.0,1 \% \mathrm{v} / \mathrm{v}\right)$

${ }_{3}^{2}$ Foi utilizado óleo mineral (Agral $\left.0,5 \% \mathrm{v} / \mathrm{v}\right)$

Ingrediente ativo

${ }^{4}$ Produto comercial 
casualizados, com quatro repetições. As parcelas foram constituídas por quatro linhas de nove plantas dispostas no espaçamento de $0,9 \mathrm{~m} \times 0,7 \mathrm{~m}$, com área útil de 7,2 $\mathrm{m}^{2}$ referente a quatorze plantas das duas linhas centrais.

A seletividade foi avaliada por meio de sintomas visuais de fitotoxicidade (Escala E.W.R.C., adaptada por Azzi \& Fernandes, 1968), aos 28, 48 e 62 dias após o plantio (DAP), número de brotações na área útil da parcela (aos 28 e 48 DAP), desenvolvimento (altura obtida pela medição a partir do nível do solo até o broto terminal de 10 plantas/parcela) aos 84 DAP e produtividade (colheita da área útil da parcela). A eficácia dos herbicidas foi avaliada pelas percentagens visuais de controle de $A$. sativa, B. pilosa, $C$. benghalensis, $R$. sativus e $S$. rhombifolia, aos 30 e 48 DAP, conforme recomendações da Sociedade Brasileira da Ciência das Plantas Daninhas (1995).

Os dados obtidos foram submetidos à análise de variância pelo teste $\mathrm{F}$ e as médias foram comparadas pelo teste de agrupamento de médias de Scott-Knott (Scott \& Knott, 1974) em nível de 5\% de probabilidade.

\section{RESULTADOS E DISCUSSÃO}

Na Tabela 3 encontram-se os dados de controle de $A$. sativa, B. pilosa e C. benghalensis aos 28 e 48 DAP. Para A. sativa, verifica-se que os controles considerados eficientes foram obtidos com os tratamentos com sulfentrazone (pré), ametryne/clomazone (pós), oxasulfuron (pós), metolachlor/ metribuzin (pré), oxyfluorfen (pós), metribuzin (pré) e ametryne isolado (pós) (controle acima de 90\%). A mistura de ametryne/diuron resultou em controle de $86 \%$ aos 48 DAP, mas não diferiu significativamente dos tratamentos considerados eficientes. $\mathrm{O}$ metolachlor não demonstrou efeito antagônico sobre o metribuzin, pois este foi eficiente no controle de aveia, tanto isolado como em mistura. Flumiclorac-pentil, trifluralin e flumioxazin, apresentaram os níveis de controle mais baixos, demonstrando que estes produtos não apresentavam ação sobre esta gramínea. Este fato explica-se porque tanto o flumiclorac-pentil quanto o flumioxazin possuem espectro de atuação basicamente sobre espécies de folhas largas. O trifluralin, utilizado normalmente para o controle de gramíneas, possui características químicas (alta volatilidade,

Tabela 3. Controle (\%) de Avena sativa, Bibens pilosa e Commelina benghalensis aos 28 e 48 DAP, na cultura da mandioca em plantio direto. Araruna, PR. 1999/2000.

\begin{tabular}{|c|c|c|c|c|c|c|c|}
\hline \multirow{2}{*}{ Tratamento } & \multirow{2}{*}{$\begin{array}{c}\text { Dose } \\
\text { (kg/ha) }\end{array}$} & \multicolumn{2}{|c|}{ - A. sativa } & \multicolumn{2}{|c|}{ B. pilosa } & \multicolumn{2}{|c|}{ C. benghalensis } \\
\hline & & 28 DAP & 48 DAP & 28 DAP & 48 DAP & 28 DAP & 48 DAP \\
\hline Testemunha capinada & - & $100 a^{1}$ & $100 \mathrm{a}$ & $100 \mathrm{a}$ & $100 \mathrm{a}$ & $100 \mathrm{a}$ & $100 \mathrm{a}$ \\
\hline Testemunha sem capina & - & $0 \mathrm{e}$ & $0 c$ & $0 \mathrm{~d}$ & $0 \mathrm{~d}$ & $0 \mathrm{e}$ & $0 \mathrm{~d}$ \\
\hline Acetochlor (pré) & 3,072 & $75 b$ & $78 \mathrm{a}$ & $88 \mathrm{a}$ & $72 b$ & $96 a$ & $94 \mathrm{a}$ \\
\hline Alachlor (pré) & 2,000 & $41 \mathrm{c}$ & $40 \mathrm{~b}$ & $41 \mathrm{c}$ & $51 \mathrm{c}$ & $56 \mathrm{c}$ & $55 \mathrm{~b}$ \\
\hline Ametryne (pré) & 1,500 & $52 \mathrm{c}$ & $44 b$ & $100 \mathrm{a}$ & $100 \mathrm{a}$ & $72 b$ & $65 b$ \\
\hline Ametryne/diuron (pré) & $0,620+0,960$ & $70 \mathrm{~b}$ & $58 \mathrm{~b}$ & $100 \mathrm{a}$ & $100 \mathrm{a}$ & $71 b$ & $80 \mathrm{~b}$ \\
\hline Flumioxazin (pré) & 0,042 & $25 \mathrm{~d}$ & $28 \mathrm{c}$ & $55 c$ & $45 c$ & $32 \mathrm{~d}$ & $35 c$ \\
\hline Isoxaflutole (pré) & 0,075 & $52 \mathrm{c}$ & $50 \mathrm{~b}$ & $100 \mathrm{a}$ & $100 \mathrm{a}$ & $69 \mathrm{~b}$ & $85 \mathrm{a}$ \\
\hline Metolachlor (pré) & 2,000 & $76 b$ & $79 \mathrm{a}$ & $10 \mathrm{~d}$ & $20 \mathrm{~d}$ & $79 b$ & $88 \mathrm{a}$ \\
\hline Metolachlor/metribuzin (pré) & $1,680+0,240$ & $95 \mathrm{a}$ & $92 \mathrm{a}$ & $100 \mathrm{a}$ & $100 \mathrm{a}$ & $98 \mathrm{a}$ & $98 \mathrm{a}$ \\
\hline Metribuzin (pré) & 0,480 & $92 \mathrm{a}$ & $92 \mathrm{a}$ & $100 \mathrm{a}$ & $100 \mathrm{a}$ & $94 \mathrm{a}$ & $97 a$ \\
\hline Oxyfluorfen (pré) & 0,480 & $51 \mathrm{c}$ & $46 b$ & $48 c$ & $24 \mathrm{~d}$ & $76 \mathrm{~b}$ & $72 b$ \\
\hline Sulfentrazone (pré) & 0,600 & $98 \mathrm{a}$ & $99 \mathrm{a}$ & $99 \mathrm{a}$ & $98 \mathrm{a}$ & $100 \mathrm{a}$ & $100 \mathrm{a}$ \\
\hline Trifluralin (pré) & 1,800 & $24 \mathrm{~d}$ & $12 \mathrm{c}$ & $0 \mathrm{~d}$ & $2 \mathrm{~d}$ & $10 \mathrm{e}$ & $15 \mathrm{c}$ \\
\hline Aclonifen (pós) & 0,900 & $74 b$ & $64 \mathrm{a}$ & $32 \mathrm{c}$ & $38 \mathrm{c}$ & $36 \mathrm{~d}$ & $24 \mathrm{c}$ \\
\hline Ametryne (pós) & 1,500 & $90 \mathrm{a}$ & $93 \mathrm{a}$ & $98 \mathrm{a}$ & $98 \mathrm{a}$ & $93 \mathrm{a}$ & $94 \mathrm{a}$ \\
\hline Ametryne/clomazone (pós) & $1,500+1,000$ & $96 a$ & $98 \mathrm{a}$ & $100 \mathrm{a}$ & $100 \mathrm{a}$ & $100 \mathrm{a}$ & $100 \mathrm{a}$ \\
\hline Ametryne/diuron (pós) & $0,620+0,960$ & $84 b$ & $86 \mathrm{a}$ & $54 \mathrm{c}$ & $92 \mathrm{a}$ & $96 \mathrm{a}$ & $95 \mathrm{a}$ \\
\hline Flumiclorac-pentil (pós) & 0,050 & $4 \mathrm{e}$ & $5 \mathrm{c}$ & $25 \mathrm{~d}$ & $25 d$ & $22 \mathrm{e}$ & $22 \mathrm{c}$ \\
\hline Isoxaflutole (pós) & 0,038 & $45 \mathrm{c}$ & $52 \mathrm{~b}$ & $71 b$ & $69 \mathrm{~b}$ & $30 \mathrm{~d}$ & $65 b$ \\
\hline Oxasulfuron (pós) & 0,045 & $96 a$ & $98 \mathrm{a}$ & $69 b$ & $99 \mathrm{a}$ & $52 \mathrm{c}$ & $72 b$ \\
\hline Oxyfluorfen (pós) & 0,480 & $94 \mathrm{a}$ & $91 \mathrm{a}$ & $58 \mathrm{c}$ & $43 c$ & $84 b$ & $88 \mathrm{a}$ \\
\hline $\mathbf{F}$ & - & $17,07^{*}$ & $15,62 *$ & $14,96^{*}$ & $17,10^{*}$ & $22,05^{*}$ & $27,89^{*}$ \\
\hline C.V. $(\%)$ & - & 23,4 & 25,8 & 28,0 & 26,3 & 20,4 & 17,0 \\
\hline
\end{tabular}

${ }^{1}$ Médias seguidas da mesma letra, em cada coluna, pertencem a um mesmo grupo, de acordo com critério de agrupamento de Scott-Knott. 
baixa solubilidade e fotodegradação) que dificultam sua utilização em áreas onde existe palhada cobrindo o solo, como no experimento em questão.

No que se refere a $B$. pilosa, os tratamentos aplicados em pré-emergência, ametryne, ametryne/diuron, metolachlor/ metribuzin, metribuzin, isoxaflutole e sulfentrazone obtiveram excelente controle (acima de 98\%). Para o acetochlor o resultado inicial foi considerado bom (87\% aos 28 DAP), mas o efeito residual não foi suficiente para proporcionar controle adequado aos $48 \mathrm{DAP}$. Em pós-emergência, o ametryne e ametryne/clomazone, apresentaram um ótimo controle (>98\%) que se manteve até aos 48 DAP. Por outro lado, os produtos oxasulfuron e ametryne/diuron proporcionaram um controle inicial baixo ( $<68 \%$ aos 28 DAP), contudo, aos 48 DAP superaram $91 \%$.

Para $C$. benghalensis, os melhores resultados em préemergência, foram obtidos com acetochlor, metolachlor/ metribuzin, metribuzin e sulfentrazone (controle acima de 94\%), seguidos de metolachlor e isoxaflutole (controle acima de $85 \%$, aos $48 \mathrm{DAP}$ ). A mistura ametryne/diuron, aos 48 DAP, proporcionou um nível de controle considerado satisfatório, mas inferior aos melhores tratamentos. Em pósemergência, os tratamentos que atingiram os melhores resul- tados de controle foram ametryne isolado e em mistura formulada com clomazone e com diuron, todos com controle acima de $94 \%$, seguidos de oxyfluorfen ( $88 \%$ de controle).

Os dados da ação dos herbicidas sobre as espécies infestantes $R$. sativus e $S$. rhombifolia, aos 28 e 48 DAP, encontram-se na Tabela 4. Para $R$. sativus os tratamentos aplicados em pré-emergência ametryne/diuron, metolachlor/ metribuzin, metribuzin, isoxaflutole e sulfentrazone, foram os produtos que mantiveram controle acima de $91 \%$ nas duas avaliações, seguidos de ametryn isolado ( $>86 \%$ de controle). O oxyfluorfen apresentou controle inicial ótimo de $94 \%$ mas, aos 48 DAP, seu índice caiu para $78 \%$ prejudicando assim seu desempenho. Os demais produtos não foram eficientes. Por outro lado, em pós-emergência, os tratamentos com aclonifen, ametryne, ametryne/clomazone, ametryne/diuron, oxasulfuron e oxifluorfen obtiveram controle $>92 \%$, aos 28 e 48 DAP.

O índice de controle de $S$. rhombifolia em pré-emergência, nos tratamentos com ametryne/diuron, metolachlor/ metribuzin e metribuzin foi excelente (100\%), seguido dos tratamentos com ametryne isolado, flumioxazin, isoxaflutole e sulfentrazone (controle superior a $88 \%$ ) não diferindo significativamente da testemunha capinada. $\mathrm{O}$ tratamento com

Tabela 4. Controle (\%) de Raphanus sativus e Sida rhombifolia aos 28 e 48 DAP, na cultura da mandioca em plantio direto. Araruna, PR. 1999/2000.

\begin{tabular}{|c|c|c|c|c|c|}
\hline \multirow{2}{*}{ Tratamento } & \multirow{2}{*}{$\begin{array}{c}\text { Dose } \\
(\mathrm{kg} / \mathrm{h} \mathbf{a})\end{array}$} & \multicolumn{2}{|c|}{ R. sativus } & \multicolumn{2}{|c|}{ S. rhombifolia } \\
\hline & & 28 DA P & 48 D A P & 28 DA P & 48 DA P \\
\hline Testemunha capinada & - & $100 \mathrm{a}^{1}$ & $100 \mathrm{a}$ & $100 \mathrm{a}$ & $100 \mathrm{a}$ \\
\hline Testemunha sem capina & - & $0 \mathrm{f}$ & $0 \mathrm{~d}$ & $0 \mathrm{~b}$ & $0 \mathrm{~d}$ \\
\hline Acetochlor (pré) & 3,072 & $52 \mathrm{~d}$ & $43 b$ & $45 \mathrm{~b}$ & $62 \mathrm{~b}$ \\
\hline Alachlor (pré) & 2,000 & $9 \mathrm{f}$ & $2 c$ & $50 \mathrm{~b}$ & $62 \mathrm{~b}$ \\
\hline Ametryne (pré) & 1,500 & $92 \mathrm{a}$ & $86 \mathrm{a}$ & $94 \mathrm{a}$ & $88 \mathrm{a}$ \\
\hline Ametryne+diuron (pré) & $0,620+0,960$ & $97 \mathrm{a}$ & $92 \mathrm{a}$ & $100 \mathrm{a}$ & $100 \mathrm{a}$ \\
\hline Flumioxazin (pré) & 0,042 & $65 \mathrm{c}$ & $58 \mathrm{~b}$ & $98 \mathrm{a}$ & $94 \mathrm{a}$ \\
\hline Isoxaflutole (pré) & 0,075 & $96 a$ & $94 \mathrm{a}$ & $80 \mathrm{a}$ & $97 \mathrm{a}$ \\
\hline Metolachlor (pré) & 2,000 & $12 \mathrm{f}$ & $15 \mathrm{c}$ & $22 b$ & $2 d$ \\
\hline Metolachlor/metribuzin (pré) & $1,680+0,240$ & $100 \mathrm{a}$ & $99 \mathrm{a}$ & $100 \mathrm{a}$ & $100 \mathrm{a}$ \\
\hline Metribuzin (pré) & 0,480 & $99 \mathrm{a}$ & $100 \mathrm{a}$ & $100 \mathrm{a}$ & $100 \mathrm{a}$ \\
\hline Oxyfluorfen (pré) & 0,480 & $94 \mathrm{a}$ & $79 a$ & $81 \mathrm{a}$ & $80 \mathrm{~b}$ \\
\hline Sulfentrazone (pré) & 0,600 & $93 \mathrm{a}$ & $93 \mathrm{a}$ & 99 a & $98 \mathrm{a}$ \\
\hline Trifluralin (pré) & 1,800 & $8 \mathrm{f}$ & $15 \mathrm{c}$ & $32 b$ & $30 c$ \\
\hline Aclonifen (pós) & 0,900 & $96 \mathrm{a}$ & $98 \mathrm{a}$ & $31 \mathrm{~b}$ & $42 c$ \\
\hline Ametryne (pós) & 1,500 & $100 \mathrm{a}$ & $97 \mathrm{a}$ & $60 \mathrm{~b}$ & $81 \mathrm{~b}$ \\
\hline Ametryne/clomazone (pós) & $1,500+1,000$ & $100 \mathrm{a}$ & $100 \mathrm{a}$ & $100 \mathrm{a}$ & $100 \mathrm{a}$ \\
\hline A metryne/diuron (pós) & $0,620+0,960$ & $99 a$ & $97 \mathrm{a}$ & $60 \mathrm{~b}$ & $78 \mathrm{~b}$ \\
\hline Flumiclorac-pentil (pós) & 0,050 & $25 \mathrm{e}$ & $15 \mathrm{c}$ & $34 b$ & $13 \mathrm{~d}$ \\
\hline Isoxaflutole (pós) & 0,038 & $54 d$ & $50 \mathrm{~b}$ & $62 \mathrm{~b}$ & $78 \mathrm{~b}$ \\
\hline Oxasulfuron (pós) & 0,045 & $84 \mathrm{~b}$. & $94 \mathrm{a}$ & $55 \mathrm{~b}$ & 93 a \\
\hline Oxyfluorfen (pós) & 0,480 & $96 \mathrm{a}$ & $92 \mathrm{a}$ & $88 \mathrm{a}$ & $85 \mathrm{~b}$ \\
\hline $\mathbf{F}$ & - & $1,04^{*}$ & $36,58 *$ & $4,68 *$ & $11,72 *$ \\
\hline C.V. (\%) & - & 13,9 & 17,7 & 42,1 & 26,9 \\
\hline
\end{tabular}

'Médias seguidas da mesma letra, em cada coluna, pertencem a um mesmo grupo, de acordo com critério de agrupamento de Scott-Knott. 
Rubem S. Oliveira Jr. et al.

oxyfluorfen, aos 28 DAP, se igualou à testemunha capinada, mas aos 48 DAP teve seu controle reduzido (80\%), sendo considerado inferior. Para o tratamento em pós-emergência com ametryne/clomazone o resultado foi excelente $(100 \%$ de controle), seguido dos tratamentos com oxasulfuron (acima de 93\%), ametryne e oxifluorfen (acima de 81\%) aos 48 DAP.

Analisando em conjunto o controle proporcionado pelos herbicidas podemos verificar, que, aos 28 e 48 DAP, metolachlor/metribuzin (pré), metribuzin (pré), sulfentrazone (pré), ametryne (pós), ametryne/clomazone (pós) apresentavam um controle eficiente para todas as espécies avaliadas, demonstrando a ótima ação dos produtos. O tratamento com ametryne isolado, em pré-emergência, resultou em bom controle de $B$. pilosa, $R$. sativus e $S$. rhombifolia, sendo superior a $86 \%$. Para ametryne/diuron e isoxaflutole (em pré) o controle das espécies investigadas foi acima de $80 \%$, com exceção de $A$. sativa. Com ametryne/diuron (pós), o controle das plantas daninhas foi superior a $86 \%$, com exceção de $S$. rhombifolia (78\%). O oxasulfuron (pós) foi eficiente para todas as espécies aos $48 \mathrm{DAP}$, exceto para $C$. benghalensis (inferior a 73\%). O oxyfluorfen (pós), também se destacou no controle de todas as espécies testadas ( $>85 \%$ de controle) com exceção da $B$. pilosa, para a qual não atingiu controle satisfatório. $\mathrm{O}$ aclonifen destacou-se no controle de $R$. sativus (acima de 97\%) e flumioxazin e oxyfluorfen (pré) no controle de S. rhombifolia.

Os dados referentes às avaliações de fitotoxicidade, estande, altura de plantas e produtividade da mandioca constam na Tabela 5. Observa-se que, na primeira avaliação, a maioria dos herbicidas causou sintomas visíveis de fitotoxicidade na parte aérea da mandioca, com exceção do acetochlor e trifluralin. Aos 48 DAP os sintomas ainda eram visíveis nos tratamentos com ametryne/diuron (pré) e metolachlor/metribuzin (pré), isoxaflutole (pré), sulfentrazone (pré) e oxasulfuron (pós). Contudo, em uma avaliação posterior (62 DAP), constatou-se que apenas no tratamento com sulfentrazone ainda havia sintomas visuais de fitotoxicidade nas plantas.

Acetochlor e trifluralin, mesmo não causando fitotoxicidade à mandioca, resultaram em uma baixa produtividade, possivelmente devido ao baixo controle sobre a maioria das espécies no início do ciclo da mandioca. Nos

Tabela 5. Valores médios de fitotoxicidade (escala E.W.C.R.) aos 28 e 48 DAP, número de manivas brotadas/12 m lineares aos 28 e 48 DAP, altura aos 84 DAP e produtividade de mandioca, em plantio direto, submetida aos diversos tratamentos. Araruna, PR. 1999/2000.

\begin{tabular}{|c|c|c|c|c|c|c|c|}
\hline \multirow{2}{*}{ Tratamento } & \multirow{2}{*}{$\begin{array}{c}\text { Dose } \\
\text { (kg/ha) }\end{array}$} & \multicolumn{2}{|c|}{ Fitotoxicidade } & \multicolumn{2}{|c|}{ Número de manivas } & \multirow{2}{*}{$\begin{array}{c}\text { Altura }(\mathrm{cm}) \\
84 \text { DAP }\end{array}$} & \multirow{2}{*}{$\begin{array}{c}\text { Produtividade } \\
\text { (kg/ha) }\end{array}$} \\
\hline & & 28 DAP & 48 DAP & 28 DAP & 48 DAP & & \\
\hline Testemunha capinada & - & 1,0 & 1,0 & $14,2 \mathrm{a}^{1}$ & $14,0 \mathrm{a}$ & $20,6 \mathrm{a}$ & $31,08 \mathrm{a}$ \\
\hline Testemunha sem capina & - & 1,0 & 1,0 & $11,2 \mathrm{a}$ & $11,0 \mathrm{~b}$ & $14,2 \mathrm{~b}$ & $22,70 c$ \\
\hline Acetochlor (pré) & 3,072 & 1,0 & 1,0 & $11,2 \mathrm{a}$ & $11,8 \mathrm{~b}$ & $17,6 \mathrm{a}$ & $19,55 c$ \\
\hline Alachlor (pré) & 2,000 & 1,5 & 1,0 & $13,5 \mathrm{a}$ & $11,2 \mathrm{~b}$ & $13,2 \mathrm{~b}$ & $19,35 \mathrm{c}$ \\
\hline Ametryne (pré) & 1,500 & 4,0 & 1,0 & $13,5 \mathrm{a}$ & $14,2 \mathrm{a}$ & $15,9 b$ & $14,25 \mathrm{~d}$ \\
\hline Ametryne/diuron (pré) & $0,620+0,960$ & 5,0 & 2,0 & $13,2 \mathrm{a}$ & $14,2 \mathrm{a}$ & $19,8 \mathrm{a}$ & $26,75 b$ \\
\hline Flumioxazin (pré) & 0,042 & 2,5 & 1,0 & $12,5 \mathrm{a}$ & $12,5 b$ & $16,5 \mathrm{a}$ & $22,50 \mathrm{c}$ \\
\hline Isoxaflutole (pré) & 0,075 & 1,5 & 5,0 & $13,8 \mathrm{a}$ & $14,5 \mathrm{a}$ & $15,9 \mathrm{~b}$ & $23,78 \mathrm{c}$ \\
\hline Metolachlor (pré) & 2,000 & 2,0 & 1,0 & $11,0 \mathrm{a}$ & $9,8 \mathrm{~b}$ & $14,5 b$ & $20,63 c$ \\
\hline Metolachlor/metribuzin (pré) & $1,680+0,240$ & 2,0 & 1,5 & $12,5 \mathrm{a}$ & $14,5 \mathrm{a}$ & $19,0 \mathrm{a}$ & $27,50 \mathrm{~b}$ \\
\hline Metribuzin (pré) & 0,480 & 2,0 & 1,0 & $12,5 \mathrm{a}$ & $14,5 \mathrm{a}$ & $20,4 \mathrm{a}$ & $26,60 b$ \\
\hline Oxifluorfen (pré) & 0,480 & 5,0 & 1,0 & $14,5 \mathrm{a}$ & $14,2 \mathrm{a}$ & $19,0 \mathrm{a}$ & $16,75 \mathrm{~d}$ \\
\hline Sulfentrazone (pré) & 0,600 & 4,5 & $5,5-$ & $11,2 \mathrm{a}$ & $13,0 \mathrm{a}$ & $12,0 \mathrm{~b}$ & $11,50 \mathrm{~d}$ \\
\hline Trifluralin (pré) & 1,800 & 1,0 & 1,0 & $12,8 \mathrm{a}$ & $11,5 \mathrm{~b}$ & $13,7 \mathrm{~b}$ & $19,18 \mathrm{c}$ \\
\hline Aclonifen (pós) & 0,900 & 5,0 & 1,0 & $14,5 \mathrm{a}$ & $14,2 \mathrm{a}$ & $17,2 \mathrm{a}$ & $30,68 \mathrm{a}$ \\
\hline Ametryne (pós) & 1,500 & 5,0 & 1,0 & $13,0 \mathrm{a}$ & $14,8 \mathrm{a}$ & $19,3 \mathrm{a}$ & $29,00 \mathrm{~b}$ \\
\hline Ametryne/clomazone (pós) & $1,500+1,000$ & 5,0 & 1,0 & $14,0 \mathrm{a}$ & $15,5 \mathrm{a}$ & $20,7 \mathrm{a}$ & $36,93 \mathrm{a}$ \\
\hline Ametryne/diuron (pós) & $0,620+0,960$ & 5,0 & 1,0 & $13,0 \mathrm{a}$ & $13,8 \mathrm{a}$ & $18,5 \mathrm{a}$ & $33,25 \mathrm{a}$ \\
\hline Flumiclorac-pentil (pós) & 0,050 & 4,5 & 1,0 & $13,8 \mathrm{a}$ & $13,2 \mathrm{a}$ & $13,8 \mathrm{~b}$ & $22,75 c$ \\
\hline Isoxaflutole (pós) & 0,038 & 5,0 & 1,0 & $13,0 \mathrm{a}$ & $13,0 \mathrm{a}$ & $17,3 \mathrm{a}$ & $27,75 b$ \\
\hline Oxasulfuron (pós) & 0,045 & 4,5 & 3,0 & $13,8 \mathrm{a}$ & $14,0 \mathrm{a}$ & $11,8 \mathrm{~b}$ & $26,88 \mathrm{~b}$ \\
\hline Oxifluorfen (pós) & 0,480 & 5,0 & 1,0 & $12,8 \mathrm{a}$ & $14,8 \mathrm{a}$ & $16,6 \mathrm{a}$ & $25,65 b$ \\
\hline $\mathbf{F}$ & - & - & - & $1,04^{\mathrm{ns}}$ & $1,99 *$ & $2,51 *$ & $6,99 *$ \\
\hline C.V. (\%) & - & - & - & 15,9 & 15,8 & 21,2 & 19,3 \\
\hline
\end{tabular}

${ }^{1}$ Médias seguidas da mesma letra, em cada coluna, pertencem a um mesmo grupo, de acordo com critério de agrupamento de Scott-Knott. 
tratamentos com sulfentrazone, ametryne e oxyfluorfen (pré), embora tenha havido controle de algumas plantas daninhas a produtividade foi inferior à testemunha sem capina. Este fato sugere que no tratamento com sulfentrazone, o período de grande fitotoxicidade causada pelo produto na cultura interferiu negativamente na produção de raízes tuberosas. Ametryne (pré) não controlou eficientemente todas as espécies de plantas daninhas, especialmente $A$. sativa e $C$. benghalensis, o que provavelmente influenciou negativamente na produtividade, mas controlou as outras infestantes e mesmo assim foi inferior à testemunha sem capina, demonstrando a fitotoxicidade. A presença de plantas daninhas no período inicial de crescimento afetou o estande da cultura, o seu crescimento inicial e a produtividade, como evidenciam os dados da testemunha sem capina (Tabela 5). Os herbicidas que demonstraram fitotoxicidade aparente na segunda avaliação não interferiram no estande da cultura. Por outro lado, os herbicidas metolachlor, alachlor, flumioxazin, acetochlor e trifluralin, aplicados em pré-emergência, não evidenciaram fitotoxicidade aparente na segunda avaliação e causaram redução no número de manivas brotadas, indicando que estes produtos podem causar dificuldades para a emergência das brotações.

A análise dos dados obtidos, em sistema de plantio direto, evidencia que as produtividades semelhantes à testemunha capinada foram obtidas com os tratamentos ametryne/ clomazone (pós) e ametryne/diuron (pós) sugerindo que, mesmo causando certo nível de injúria nas plantas de mandioca, o controle inicial das plantas daninhas e a fitotoxicidade apenas inicial possibilitou a cultura recuperar-se e produzir normalmente. As maiores reduções no rendimento de raízes estiveram associadas aos tratamentos onde foram aplicados os herbicidas em pré-emergência, seja pela prolongada fitotoxicidade do tratamento (sulfentrazone) ou pelo insuficiente controle de algumas plantas daninhas presentes na área (oxyfluorfen e ametryne), confirmando os experimentos de Doll \& Piedrahita (1974, 1976), Oliveira Jr. (1994), Oliveira Jr. et al. (1995).

\section{CONCLUSÕES}

A mistura ametryne/clomazone, utilizada em pósemergência, a 1,50/1,00 kg/ha, foi excelente no controle de todas as plantas daninhas no experimento.

A mistura ametryne/diuron, em pós-emergência, a 0,62/0,96 kg/ha, foi eficiente no controle de A. sativa, $B$. pilosa, C. benghalensis e $R$. sativus.

As misturas ametryne/clomazone e ametryne/diuron, aplicados em pós-emergência, foram seletivas à cultura de mandioca e não afetaram a produtividade.

$\mathrm{O}$ aclonifen em pós-emergência foi seletivo à cultura da mandioca.

Metolachlor/metribuzin $(1,68 / 0,24 \mathrm{~kg} / \mathrm{ha})$ e metribuzin $(0,48 \mathrm{~kg} / \mathrm{ha})$, aplicados em pré-emergência, e ametryne (1,50 $\mathrm{kg} / \mathrm{ha}$ ), em pós-emergência, proporcionaram excelente con- trole das plantas daninhas presentes na área do experimento, mas reduziram significativamente a produção de raízes.

Ametryne, oxyfluorfen e sulfentrazone, aplicados em pré-emergência, não foram seletivos a cultura da mandioca, sendo portanto, desaconselhável seu uso.

Acetochlor (pré-emergência), alachlor (pré-emergência), flumioxazin (pré-emergência), metolachlor (pré-emergência), trifluralin (pré-emergência) e flumiclorac-pentil (pósemergência), apesar do baixo nível de controle, foram considerados seletivos à cultura da mandioca.

\section{LITERATURA CITADA}

ALCÂNTARA, E.N.; CARVALHO, J.E.B.; LIMA, P.C. Determinação do período crítico de competição das plantas daninhas com a cultura da mandioca (Manihot esculenta Crantz). In: EPAMIG. Projeto Mandioca, relatório 76/79. Belo Horizonte: EPAMIG, 1982. p.147149.

ALCÂNTARA, E.N.; LIMA, P.C. Efeito de doses de herbicida para a cultura da mandioca (Manihot esculenta Crantz). In: EPAMIG. Projeto Mandioca, relatório 76/79. Belo Horizonte: EPAMIG, 1982. p.130-135.

ALCÂNTARA, E.N.; SOUZA, I.F. Herbicidas na cultura da mandioca. In: EPAMIG. Projeto Mandioca, relatório 76/79. Belo Horizonte: EPAMIG, 1982. p.136-141.

ALVES, A.A.C. Fisiologia da mandioca. In: CURSO NACIONAL DE MANDIOCA, 7. Cruz das Almas, 1990. Embrapa-CNPNF, 1990. p.25.

AZZI, G.M.; FERNANDES, J. Método de julgamento do efeito herbicida. In: CONGRESSO BRASILEIRO DE HERBICIDAS E ERVAS DANINHAS, 6. Sete Lagoas, 1966. Anais... Sete Lagoas: SBHED, 1968. p.21-29.

CARVALHO, J.E.B.; CALDAS, R.C.; COSTA NETO, A.O.; CARDOSO, S.S.; MASCARENHAS, L.; BARBOSA, C.V. Período crítico de competição das plantas daninhas com a cultura da mandioca em um ecossistema do nordeste brasileiro. Revista Brasileira de Mandioca, v.12, p.85-93, 1993.

CARVALHO, J.E.B.; QUEIROZ, G.M.; LYRA FILHO, H.P.; ALVES, A.A.C.; CALDAS, R.C.; REZENDE, G.O.; PEREIRA, R.C.A. Período crítico de competição das plantas daninhas com a cultura da mandioca em três ecossistemas do nordeste brasileiro. Revista Brasileira de Mandioca, v.9, p.29-40, 1990.

CONCEIÇÃO, A.J. A mandioca. Cruz das Almas: UFBA/ EMBAPA/BNB/BRASCAN NORDESTE, 1979. 382p. 
DOLL, J.D.; PIEDRAHITA, W. Margem de seletividade de vários herbicidas en yuca (Manihot esculenta Crantz). Rev. Comalfi, v.1, n.1, p.14-19, 1974.

DOLL, J.D.; PIEDRAHITA, W. Metodos de control de malezas em yuca. Centro Internacional de Agricultura Tropical, 1976. 12 p.

FAO. Rome. Production yearbook, v.45, p.5-95, 1996.

FERREIRA, T.N.; FARIAS, A.D. Expansão do sistema de plantio direto no Rio Grande do Sul. Porto Alegre: EMATER-RS, 1997. (Informativo da EMATER-RS. Série Solos, v.8, n.4).

FORNAROLLI, D.A.; RODRIGUES, B.N.; LIMA, J.; VALÉRIO, M.A. Influência da cobertura morta no comportamento do herbicida atrazine. Planta Daninha, v.16, n.2, p.97-107, 1998.

HOWELER, R.H., BALLESTEROS, D. EI cultivo de la yuca en los Ilanos Orientales de Colombia: variedades y prácticas agronómicas. Cali: CIAT, 1987.29p. (Boletim Técnico, 35).

LORENZI, J.O.; DIAS, C.A. Cultura da mandioca. Campinas: CATI, 1993. 41p. (Boletim Técnico, 211).

MIRANDA, I.J.; LAVINA, M.L.; POA, A.C. Controle de plantas daninhas na cultura da mandioca através de herbicidas pré-emergentes pós plantio em podzólico vermelho amarelo distrófico. In: CONGRESSO BRASILEIRO DE HERBICIDAS E PLANTAS DANINHAS, 20. Florianópolis, 1995. Resumos... Florianópolis: SBCPD, 1995. p.138-139.

OLIVEIRA JR., R.S. Seletividade e eficiência de trifluralin e diuron aplicados em diferentes formas na cultura da mandioca (Manihot esculenta Crantz). Revista Unimar, v.16, n.2, p.317-325, 1994.

OLIVEIRA JR., R.S.; ZOTARELLI, L.; HIRAI, L.T. Seletividade e eficiência de controle de clethodim na fase inicial de desenvolvimento da cultura da mandioca. In: CONGRESSO BRASILEIRO DE HERBICIDAS E PLANTAS DANINHAS, 20. Florianópolis, 1995. Resumos... Florianópolis: SBCPD, 1995. p.166-167.

PERESSIN, V.A. Matointerferência na cultura da mandioca (Manihot esculenta Crantz) em duas regiões do estado de São Paulo. Jaboticabal: Universidade Estadual Paulista, 1997. 132p. (Tese de Doutorado).

PINHO, J.L.N.; QUEIROZ, G.M.; MELO, F.L.O.; LOPES, J.G.V.; OLIVEIRA, F.C. Controle de plantas daninhas na cultura da mandioca (Manihot esculenta Crantz), no Ceará. In: EPACE. Relatório anual de pesquisa da EPACE. Fortaleza, CE. 1980. p.53-81.

RODRIGUES, B.N. Influência da cobertura morta no comportamento dos herbicidas imazaquin e clomazone. Planta Daninha, v.11, n.1/2, p.21-28, 1993.

SCOTT, A.; KNOTT, M. Cluster-analysis method for grouping means in analysis of variance. Biometrics, v.30, n.3, p.507-512, 1974.

SOCIEDADE BRASILEIRA DA CIÊNCIA DAS PLANTAS DANINHAS. Procedimentos para instalação, avaliação e análise de experimentos com herbicidas. Londrina: SBCPD, 1995. p.42.

WARON, L.A.; GARTNER, J.J. El cultivo de la yuca. In: Instituto Colombiano Agropecuário. Centro Nacional de Investigaciones Agropecuarias de Palmira, Colombia, 1972. p.14. 